\title{
Silicon Sensors for High-Luminosity Trackers - RD50 Collaboration status report
}

\author{
Susanne Kuehn *广 \\ Albert-Ludwigs-Universität Freiburg (DE) \\ E-mail: susanne.kuehn@cern.ch
}

\begin{abstract}
The revised schedule for the Large Hadron Collider (LHC) upgrade foresees a significant increase of the luminosity of the LHC by upgrading towards the HL-LHC (High Luminosity-LHC). The final upgrade is planned for around the year 2023, followed by the HL-LHC running. This is motivated by the need to harvest the maximum physics potential from the machine. It is clear that the high integrated luminosity of $3000 \mathrm{fb}^{-1}$ will result in very high radiation levels, which manifest a serious challenge for the detectors. This is especially true for the tracking detectors installed close to the interaction point. For HL-LHC, all-silicon central trackers are being studied in ATLAS, CMS and LHCb, with extremely radiation hard silicon sensors to be employed in the innermost layers. Within the RD50 Collaboration, a massive R\&D program is underway, with an open cooperation across experimental boundaries to develop silicon sensors with sufficient radiation tolerance. This report presents several research topics of the collaboration and highlights some recent results. Emphasize is given on results of sensors made from p-type silicon bulk, which have superior radiation hardness as they collect electrons instead of holes. A further area of activity is the development of advanced sensor types like active edges, thin, charge multiplication and 3D detectors. Results are shown from several detector technologies and silicon materials at radiation levels corresponding to HL-LHC fluences. Based on the results, recommendations are given for the silicon detectors to be used for LHC detector upgrades.
\end{abstract}

Technology and Instrumentation in Particle Physics 2014,

2-6 June, 2014

Amsterdam, the Netherlands

\footnotetext{
*Speaker.

† On behalf of the RD50 Collaboration, http://cern.ch/rd50.
} 


\section{Introduction}

For the envisaged upgrade of the LHC to the HL-LHC a significant increase of the LHC design luminosity is foreseen and an integrated luminosity of $3000 \mathrm{fb}^{-1}$ is planned [1]. The upgrade is foreseen to start in about the year 2023 and a severe radiation dose will occur for the inner detector layers for the silicon tracking detectors in the LHC experiments ATLAS, CMS and LHCb. For ATLAS and CMS doses of about $1 \times 10^{16} 1-\mathrm{MeV}$-equivalent neutrons $\left(\mathrm{n}_{\mathrm{eq}}\right) / \mathrm{cm}^{2}$ are expected for the pixel detector layers. They occur mainly due to charged hadrons. For the strip detectors at radii of $40 \mathrm{~cm}$ to $100 \mathrm{~cm}$ from the interaction point, radiation damage is expected mainly due to neutrons with doses of about $1 \times 10^{15} \mathrm{n}_{\text {eq }} / \mathrm{cm}^{2}$ [2]. To facilitate efficient and well-performing tracking detectors, the investigation and understanding of radiation damage of silicon sensors is essential. This is the key task of the RD50 Collaboration. It has studied before and after irradiation specific research fields, namely defect material characterization, detector characterization, new structures and full detector systems. Most of the 275 RD50 members from 50 institutions are as well members of one of the LHC experiments. The collaboration arranges common sensor production runs, two workshops per year and access to irradiation facilities.

In the following selected recent results of the research fields of the RD50 collaboration will be presented and details are given for possible silicon sensors for the upgrade of the LHC experiments.

\section{Defect characterization}

The main goal of the research field defect characterization is the identification of defects causing changes of detector properties, namely trapping, leakage current and the effective doping concentration, the depletion voltage respectively. The generation of defects in the band gap depends on the incident particle energy and type. Increase of knowledge is used for device engineering and gives input to simulations. The defect parameters like cross section, ionization energy, concentration, and type are important to know. Various techniques are used for the defect analysis before and after irradiation, e.g. thermally stimulated current technique, deep level transient spectroscopy or capacity and leakage current measurements versus depletion voltage. An exemplary result measuring defects after irradiation with electrons is shown in figure 1(a). It gives the dependence of the defects on the incident electron energy for float zone sensors [3]. Some defects increase the leakage current. It scales independently of material with $\alpha=4.38 \times 10^{-17} \mathrm{~A} / \mathrm{cm}^{2}$ up to fluences of $2 \times 10^{15} \mathrm{n}_{\mathrm{eq}} / \mathrm{cm}^{2}$ [4]. Moreover, the leakage current decreases after annealing and higher annealing temperatures lead to a further reduction [5]. Deep level transient spectroscopy measurements attribute this to the defects E4/E5 and E205a.

A consistent set of defects has been found after irradiation with different particles. The defect introduction rates were measured and details are e.g. given in [6].

\section{Device simulations}

A simulation group has recently been formed and involves several institutes. Simulations are essential to predict electric fields and trapping in silicon sensors. The goal is to develop an approach for simulation of performance of irradiated silicon detectors using professional software. Effective 


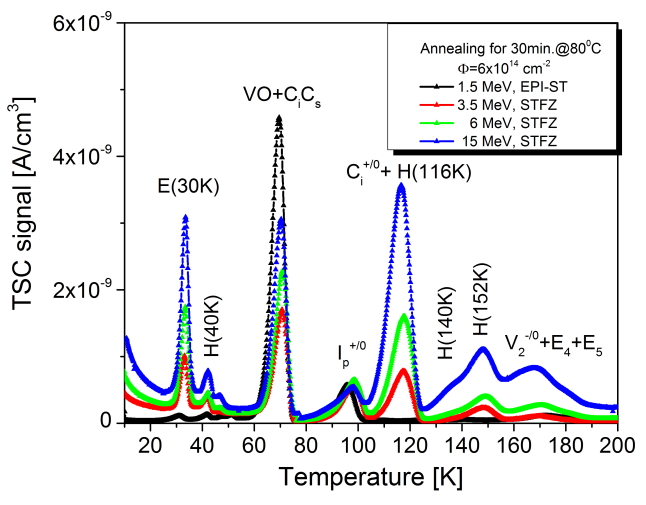

(a)

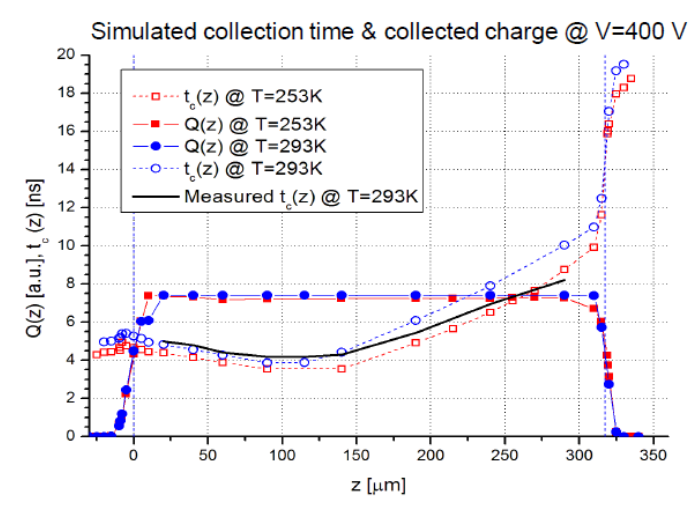

(b)

Figure 1: In (a) thermally stimulated current signal of float zone sensors after irradiation with electrons of different kinetic energies to doses of $6 \times 10^{14} \mathrm{n}_{\mathrm{eq}} / \mathrm{cm}^{2}$. In (b) simulated and measured collection time and collected charge of an unirradiated p-in-n sensor. The detector front electrode at $0 \mu \mathrm{m}$ and back electrode at $320 \mu \mathrm{m}$ are indicated by dashed lines.

mid gap levels (a deep acceptor and a deep donor level) are used. The large parameter space is tuned by experimental data. They start to have predictive power as can be seen in figure 1(b) [7]. It shows for an unirradiated n-type sensor the collection time and collected charge versus depth of charge deposition in the detector for simulation and data taken with the edge-TCT method. A good agreement is found. Also leakage current, depletion voltage, the charge collection efficiency and partially trapping of irradiated sensors can be modelled. Currently, a common database with cross sections and concentrations is in preparation and more tuning to data is ongoing.

\section{Full detector systems}

The research field of full detector systems evaluates systematically strip and pixel sensors which are connected to fast electronics and measured before and after irradiation with protons, neutrons and pions.

\subsection{Planar detectors}

One highly performing detector type are planar p-type detectors. They are currently the baseline for the upgrade of the ATLAS and CMS experiment's silicon strip tracking detectors. A comparison of the collected charge for different planar detectors after irradiation is shown in figure 2. $\mathrm{N}$-in-p sensors collect more than 5000 electrons and are fully operational until highest fluences of $1 \times 10^{16} \mathrm{n}_{\text {eq }} / \mathrm{cm}^{2}$. P-in- $n$ devices collect a smaller amount of charge $[8,9,10]$. The $n$-in-p sensors have a superior performance due to no space charge inversion, a favourable combination of weighting and electric field after irradiation and the readout at n-type electrodes, resulting in a fast collection of electrons and shorter trapping times. It can also be seen that operation at higher bias voltages can enhance the amount of collected charge. Within the RD50 Collaboration this effect, 
called charge multiplication, has been observed and the curve of the p-type detector (biased to $1700 \mathrm{~V}$ ) indicates that after irradiation more charge is collected than before [11].

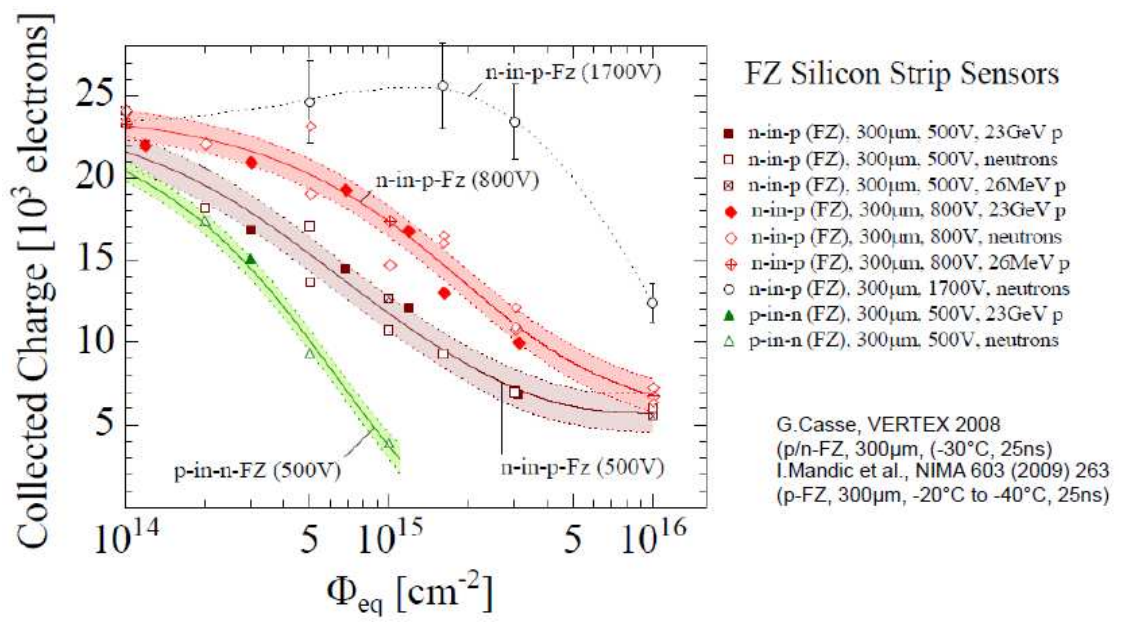

Figure 2: Collected charge of different planar float zone detectors after irradiation to fluences of up to $1 \times 10^{16} \mathrm{n}_{\mathrm{eq}} / \mathrm{cm}^{2}$.

\subsection{Float zone and magnetic Czochralski detectors}

In order to reach an improved radiation tolerance of p-type detectors, both float zone and magnetic Czochralski (MCz) sensors are investigated. $\mathrm{MCz}$ material has a higher oxygen concentration (about $5 \times 10^{17} \mathrm{~cm}^{-3}$ ) and was introduced by the RD50 Collaboration. Figure 3(a) displays the collected charge for float zone (FZ) and $\mathrm{MCz}$ as a function of annealing time at room temperature. The results for $200 \mu \mathrm{m}$ thick p-type sensors after mixed irradiation to $1.5 \times 10^{15} \mathrm{n}_{\mathrm{eq}} / \mathrm{cm}^{2}$ give a higher collected charge for $\mathrm{MCz}$ sensors [12]. The $\mathrm{MCz}$ sensors are less affected by annealing and show

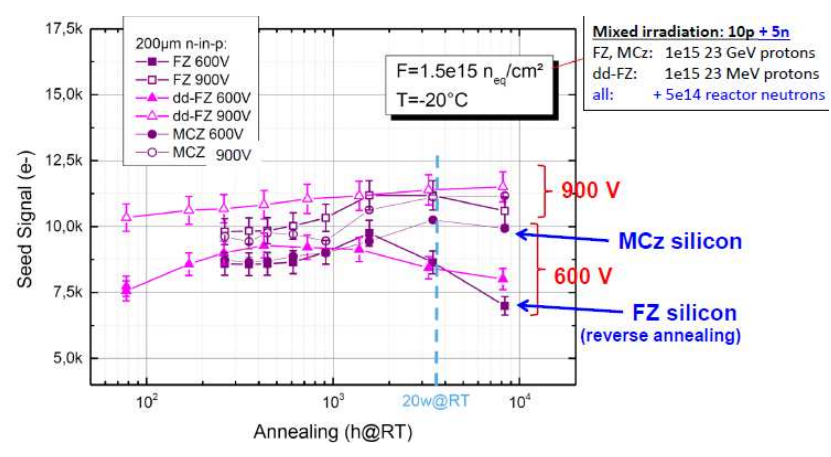

(a)

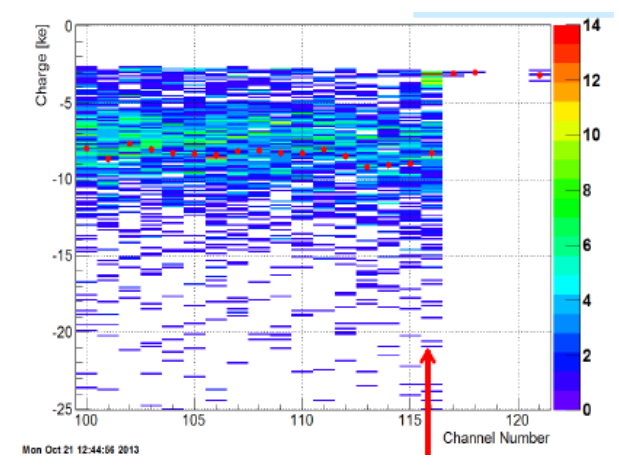

(b)

Figure 3: In (a) collected charge of different planar float zone and MCz detectors after irradiation to fluences of $1.5 \times 10^{15} \mathrm{n}_{\mathrm{eq}} / \mathrm{cm}^{2}$ for different annealing times. In (b) charge of slim edge planar p-type strip sensor after irradiation to fluence of $4 \times 10^{15} \mathrm{n}_{\mathrm{eq}} / \mathrm{cm}^{2}$. The mean charge is indicated with red circles. The arrow marks the edge strip. 
a stable annealing behaviour. Also an improved performance in mixed fields due to compensation of neutron and charged particle damage in oxygen rich $\mathrm{MCz}$ is observed.

\subsection{Thin detectors}

Moreover, thin detectors are investigated. The collected charge of several sensors of various thicknesses was measured after proton irradiation to $1 \times 10^{15} \mathrm{n}_{\mathrm{eq}} / \mathrm{cm}^{2} .100-150 \mu \mathrm{m}$ thick sensors have shown highest collected charge compared to thicker samples at moderate bias voltages (200$300 \mathrm{~V})$ [13]. Thin sensors are additionally advantagous due to their reduced amount of material.

\section{New structures}

The third research field covers new structures. Beside the already mentioned thin detectors, a variety of new devices like 3D detectors, charge multiplication sensors and slim or active edge sensors are under study.

\subsection{D detectors}

3D sensors have doped columns vertically to the surface etched into the silicon bulk. This geometry decouples the depletion voltage and detector thickness (collected charge), resulting in a higher radiation resistance [14]. Several manufacturers are able to produce devices even in doublesided technology $[15,16,17]$. Since these detectors can be produceddd in large numbers and give a decent signal after irradiation to doses of $1 \times 10^{16} \mathrm{n}_{\mathrm{eq}} / \mathrm{cm}^{2}, 25 \%$ of the sensors of the recently installed insertable b-layer of the ATLAS experiment are 3D sensors [18].

Unfortunately, the layout leads to low-field regions, resulting in inefficiencies. Sensors overcoming this feature are 3D trench electrode detectors which have a concentric trench electrode surrounding the central hexagonal signal collecting column electrode $[19,20]$. The hexagonal electrode pattern leads to an uniform electric field. First p-type devices with $300 \mu \mathrm{m}$ thickness have been produced and tested. The full depletion voltage is about $95 \mathrm{~V}$ after irradiation to $1 \times$ $10^{16} \mathrm{n}_{\mathrm{eq}} / \mathrm{cm}^{2}$ and all charge is collected in tests with an ${ }^{241}$ Americium source. In a next step position-resolved charge collection measurements are planned with a laser setup.

\subsection{Active and slim edge detectors}

A beneficial feature for silicon sensors' use in tracking detectors is a reduced edge width. Several methods were developped to minimize the non-active volume, resulting in slim or active edge sensors. The scribe-cleave-passivate technology leads to $150 \mu \mathrm{m}$ slim edges compared to edge widths larger than $500 \mu \mathrm{m}$ [21]. Charge collection measurements of p-type strip sensors with $285 \mu \mathrm{m}$ thickness and after irradiation to $4 \times 10^{15} \mathrm{n}_{\mathrm{eq}} / \mathrm{cm}^{2}$ show, as seen in figure $3(\mathrm{~b})$, a similar median charge for strips close to the edge and to other strips [22]. Alternatively, pixel sensors with active edges are manufactured by cutting the edges down to $50 \mu \mathrm{m}$ width and doping trenches by four-quadrant implementation [13]. The overall efficiency, leakage current and noise is measured to be unaffected for these thin pixel sensors with $125 \mu \mathrm{m}$ width edges even after irradiation to $1 \times 10^{15} \mathrm{n}_{\mathrm{eq}} / \mathrm{cm}^{2}$. 


\section{Charge multiplication effect}

The collected charge of full detector systems is already shown in figure 2, where at high bias voltages, the effect of charge multiplication is observed. It was measured in several devices, namely strip sensors, 3D sensors and diodes [23, 24, 25]. Its origin is understood to come from a high negative space charge concentration in the bulk after irradiation. The resulting increased electric field close to n-type strips leads to impact ionization. Goals in the research on charge multiplication sensors are beside simulation and prediction of the effect, also measurements of long term signal and noise behaviour. A dedicated program has been setup within the RD50 Collaboration to understand and optimize the mechanism and to evaluate its exploitation.

\subsection{Sensors with enhanced charge multiplication}

First, sensors with trenches were produced. They have 5 to $50 \mu \mathrm{m}$ deep and $5 \mu \mathrm{m}$ wide trenches below the strips. This modification to usual sensor designs should increase the electric field close to the strips. Measurements after irradiation give both for 5 and $50 \mu \mathrm{m}$ trenches an enhanced collected charge [26, 27].

Second, p-type sensors with different strip widths and pitches were manufactured. In addition, the diffusion time and implant energy was variied. The effect of charge multiplication is seen on the $300 \mu \mathrm{m}$ thick strip sensors at bias voltages above $600 \mathrm{~V}$ for sensors with width-over-pitch ratios of about 0.1 [28]. Currently, the long term behaviour is under test and first results tend to show a degradation after several days of running.

Third, newly developped charge multiplication sensors with an implemented multiplication layer, a deep $\mathrm{p}^{+}$implant are under study in a RD50 project [29]. A schematic drawing of a socalled low gain avalanche diode is given in figure 4. A p-doped diffusion layer is below the cathodes, leading to a multiplication layer. An edge termination is needed which is done with a low doping n-well [30]. Several devices were produced and charge multiplication measurements show

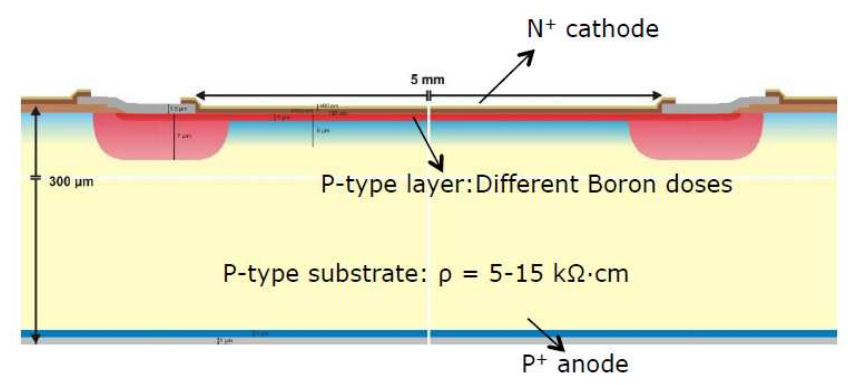

Figure 4: Schematic drawing of low gain avalanche diode.

a collected charge of more then 200,000 electrons after full depletion [31]. Gain values of about 10 are measured. Also transient current technique measurements show the occurence of the multiplication effect. After irradiation to doses of $2 \times 10^{15} \mathrm{n}_{\text {eq }} / \mathrm{cm}^{2}$ a reduction in gain is occuring. Values low as 1.5 are measured, showing a degradation of the multiplication effect [32]. The reasons are under investigation and trapping doesn't seem to be the cause but a boron removal in the highly 
doped p-type layer which leads to an reduction of the electric field. Currently more wafers are in production for detailled tests and an irradiation programme is planned.

\section{Summary}

Several results were presented of the wide R\&D program of the RD50 Collaboration. The following achievements were reached in light of the LHC experiments. For inner layers of the silicon trackers, exposed to fluences of up to $1 \times 10^{16} \mathrm{n}_{\mathrm{eq}} / \mathrm{cm}^{2}$ planar sensors are found to collect a decent amount of charge and have hit efficiencies of about 97\%. N-in-p (or n-in-n) material is the candidate material, since it is important to collect electrons. Thin detectors overcome the requirement of high bias voltages and are advantageous at doses above $1 \times 10^{15} \mathrm{n}_{\mathrm{eq}} / \mathrm{cm}^{2}$. 3D detectors are an alternative option and show good performance requiring lower bias voltages. For the outer layer with fluences of about $2 \times 10^{15} \mathrm{n}_{\mathrm{eq}} / \mathrm{cm}^{2}$ planar $\mathrm{n}$-in-p sensors are the baseline for the ATLAS and CMS upgrade strip tracker. Since float zone material has a shorter reverse annealing at room temperature compared to $\mathrm{MCz}, \mathrm{MCz}$ is also an option due to its compensation of damage in mixed fluences.

The recent key results of the RD50 Collaboration are that a lot of progress has been achieved on the understanding of microscopic defects and the origin of positive space charge. A simulation working group has been formed and simulations get predictive power. Many new structures are under investigation and a consolidation of data on n-in-p-type detectors, thin segmented and slim or active edge sensors has been accomplished. The effect of charge multiplication is investigated systematically to allow its exploitation and dedicated sensors are produced. For many further results aiming for radiation hard semiconductor devices for future colliders, it is referred to the website of the RD50 Collaboration http://www.cern.ch/rd50/.

\section{Acknowledgment}

The author would like to thank the colleagues of the RD50 Collaboration for material and support.

\section{References}

[1] L. Rossi and O. Brüning, High Luminosity Large Hadron Collider - A description for the European Strategy Preparatory Group, CERN-ATS-2012-236.

[2] I. Dawson, Radiation predictions at the SLHC and irradiation facilities, presented at the ATLAS Tracker Upgrade Workshop, Liverpool, UK, Dec. 6-8, 2006.

[3] R. Radu, et al., Radiation Damage in n-type silicon diodes after electron irradiation with energies between 1.5 MeV and $15 \mathrm{MeV}$ Nucl. Instrum. Meth. A 730 (2013) 84-90.

[4] H. Neugebauer, Systematic investigation of p-irradiated Micron pad detectors of different silicon materials, presented at the $23^{\text {rd }}$ RD50 Workshop, Nov. 2013.

[5] A. Junkes, Status of defect investigations, in proceedings of Vertex 2011, PoS (Vertex 2011) 035.

[6] I. Pintilie et al., Radiation-induced point- and cluster-related defects with strong impact on damage properties of silicon detectors, Nucl. Instrum. Meth. A 611 (2009) 52-68. 
[7] T. Peltola, Non-uniform 3-level defect model and status of edge-TCT simulations, presented at the $23^{\text {rd }}$ RD50 Workshop, Nov. 2013.

[8] M. Moll, Recent advances in the development of radiation tolerant silicon detectors for the Super-LHC, Astroparticle, Particle and Space Physics, Detectors and Medical Physics Applications, proceedings of the 11th Conference, Villa Olmo, Como, Italy, C. Leroy, ed., World Scientific Publishing, 2010, 101-110.

[9] S. Kuehn, Charge Collection measurements of n-in-p strip detectors after mixed irradiation to HL-LHC fluences and annealing, presented at the 21 Rt RDO Workshop, Nov. 2012.

[10] G. Casse, et al., Measurements of charge collection efficiency with microstrip detectors made on various substrates after irradiations with neutrons and protons with different energies, in proceedings of Vertex 2008, PoS (Vertex 2008) 036.

[11] I. Mandic, et al., Measurement of anomalously high charge collection efficiency in $n^{+} p$ strip detectors irradiated by up to $1 \times 10^{16} \mathrm{n}_{\mathrm{eq}} / \mathrm{cm}^{2}$, Nucl. Instrum. Meth. A 603 (2009) 263-267.

[12] G. Steinbrück, Radiation hard silicon sensors for the CMS tracker upgrade, presented at the $23^{\text {rd }}$ RD50 Workshop, Nov. 2013.

[13] S. Terzo, Irradiated n-in-p planar pixel sensors of different thicknesses and active edge designs, presented at the $23^{\text {rd }}$ RD50 Workshop, Nov. 2013.

[14] S. I. Parker, C. J. Kenney and J. Segal, 3D - A proposed new architecture for solid-state radiation detectors, Nucl. Instrum. Meth. A 395 (1997) 328-343.

[15] G. Pellegrini, et al., First double-sided 3-D detectors fabricated at CNM-IMB, Nucl. Instrum. Meth. A 592 (2008) 38-43.

[16] E. Vianello, et al., Optimization of double-side 3D detector technology for first productions at FBK, IEEE NSS Conf. Record, N10-6, 2011.

[17] G.-F. Dalla Betta et al., Performance evaluation of 3D-DDTC detectors on p-type substrates, Nucl. Instrum. Meth. A 624 (2010) 459-464.

[18] The ATLAS Collaboration, ATLAS Insertable B-Layer Technical Design Report Addendum, CERN, Geneva, May, 2012, CERN-LHCC-2012-009, ATLAS-TDR-019-ADD-1, Addendum to CERN-LHCC-2010-013, ATLAS-TDR-019.

[19] Z. Li, New BNL 3D-Trench electrode Si detectors for radiation hard detectors for 5 LHC and for $X$-ray applications, Nucl. Instrum. Meth. A 658 (2011) 90-97.

[20] A. Montalbano, A systematic study of BNL's 3D-Trench Electrode detectors, http://dx.doi.org/10.1016/j.nima.2014.03.066.

[21] V. Fadeyev, Scribe-cleave-passivate (SCP) slim edge technology for silicon sensors, Nucl. Instrum. Meth. A 731 (2013) 260-265.

[22] V. Fadeyev, Status of Scribe-Cleave-Passivate (SCP) Slim Edge Technology, presented at the $23^{\mathrm{rd}}$ RD50 Workshop, Nov. 2013.

[23] G. Casse, et al., Enhanced efficiency of segmented silicon detectors of different thicknesses after proton irradiations up to $1 \times 10^{16} \mathrm{n}_{\mathrm{eq}} / \mathrm{cm}^{2}$, Nucl. Instrum. Meth. A 624 (2011) 401-404.

[24] M. Koehler, et al., Comparative measurements of highly irradiated $n$-in-p and $p$-in- $n$ 3D silicon strip detectors, Nucl. Instrum. Meth. A 659 (2011) 272-281. 
[25] J. Lange, Charge Multiplication in Highly-Irradiated Epitaxial Silicon Diodes, presented at the $16^{\text {th }}$ RD50 Workshop, June 2010.

[26] G. Casse, et al., Charge multiplication in irradiated segmented silicon detectors with special strip processing, Nucl. Instrum. Meth. A 699 (2013) 9-13.

[27] P. Fernández-Martínez, et al., Simulation of new p-type strip detectors with trench to enhance the charge multiplication effect in the n-type electrodes, Nucl. Instrum. Meth. A 658 (2011) 98-102.

[28] C. Betancourt, Charge Collection Measurements on Dedicated RD50 Charge Multiplication SSDs, presented at the $23^{\text {rd }} R D 50$ Workshop, Nov. 2013.

[29] G. Pellegrini et al., Technology developments and first measurements of Low Gain Avalanche Detectors (LGAD) for High Energy Physics applications, accepted by Nucl. Instrum. Meth. A

[30] M. Baselga, Fabrication of new p-type pixel detectors with enhanced multiplication effect in the $n$-type electrodes, presented at the $8^{\text {th }}$ Trento Workshop on Advanced Silicon Radiation Detectors $(3 D$ and p-type), Feb. 2013.

[31] G. Kramberger, Studies of CNM diodes with gain, presented at the $22^{\text {nd }}$ RD50 Workshop, June 2013.

[32] G. Kramberger, Studies of LGAD diodes in Ljubljana (an update), presented at the $23^{\text {rd }}$ RD50 Workshop, Nov. 2013. 